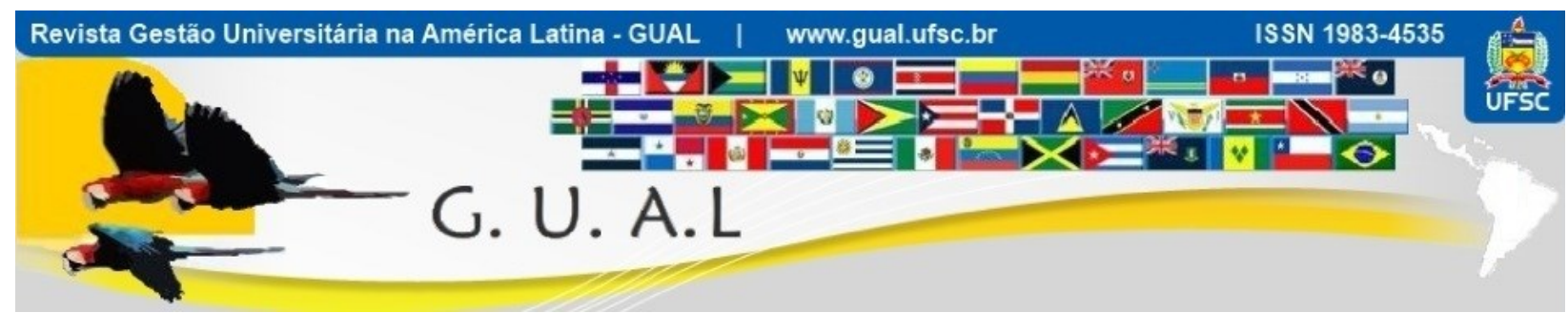

DOI: http://dx.doi.org/10.5007/1983-4535.2017v10n2p274

\title{
RELAÇÕES INTERORGANIZACIONAIS COMO LÓCUS DE VANTAGEM COMPETITIVA: MANIFESTAÇÕES EM INSTITUIÇÕES BRASILEIRAS DE EDUCAÇÃO SUPERIOR
}

\author{
INTERORGANIZATIONAL RELATIONS AS LOCUS OF COMPETITIVE \\ ADVANTAGE: MANIFESTATIONS IN BRAZILIAN INSTITUTIONS OF HIGHER \\ EDUCATION
}

Fábio Dal-Soto, Doutorando Universidade de Cruz Alta - UNICRUZ e Universidade do Vale do Rio dos Sinos - UNISINOS dalsoto.gel@terra.com.br

Jefferson Marlon Monticelli, Doutorando Universidade do Vale do Rio dos Sinos - UNISINOS jeffmarlon@hotmail.com

Ingridi Vargas Bortolaso, Doutora Universidade de Santa Cruz do Sul - UNISC ingridibortolaso@unisc.br

Suelen Geíse Telocken, Mestranda Universidade Federal de Santa Maria - UFSM sutelocken@hotmail.com

Recebido em 18/dezembro/2015

Aprovado em 05/março/2017

Sistema de Avaliação: Double Blind Review

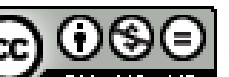

Esta obra está sob uma Licença Creative Commons Atribuição-Uso. 


\title{
RESUMO
}

Os relacionamentos interorganizacionais têm se constituído como importante lócus na geração de diferenciais competitivos e, consequentemente, uma interessante unidade de análise para a compreensão da vantagem competitiva. A partir disso, o objetivo deste artigo é analisar o desenvolvimento dessas relações como fontes de vantagens competitivas, por meio do Consórcio das Universidades Comunitárias Gaúchas (COMUNG), composto por 15 instituições comunitárias de educação superior (ICES), localizado no sul do Brasil. Trata-se, portanto, de um estudo de caso único com múltiplas unidades de análise, realizado por meio de entrevistas in loco com o Presidente do Consórcio e os gestores das ICES pesquisadas, além de dados secundários. Os resultados mostram um conjunto de ações realizadas por meio do COMUNG como fontes de vantagens relacionais, como a movimentação política conjunta, o compartilhamento de experiências administrativas e acadêmicas e o estabelecimento de acordos tácitos de competição. Logo, a posição de insider no COMUNG oportuniza às ICES o aprendizado de forma mais rápida, a redução e o compartilhamento de custos, a salvaguarda das áreas geográficas de competição e a qualificação e diferenciação das atividades em geral.

Palavras-chave: Relações interorganizacionais. Vantagem competitiva. Educação superior.

\begin{abstract}
Interorganizational relationships have been established as important locus of generating competitive advantages and consequently an interesting analysis unit for understanding the competitive advantage. From this, the aim of this paper is to analyze the development of these relations as sources of competitive advantages, through the Consortium of Community Universities Gaucho (COMUNG), composed of 15 community institutions of higher education, located in southern Brazil. Thus, it's a single case study with multiple units of analysis, accomplished through in loco interviews with the President of the Consortium and the managers of the researched institutions, as well as secondary data. The results show a set of actions accomplished by COMUNG as sources of relational advantages, such as joint political movement, sharing administrative and academic experiences and the establishment of tacit agreements of competition. Therefore, the insider position in COMUNG gives opportunity to institutions learning faster, reducing costs and sharing, the safeguarding geographical areas of competition and the qualification and differentiation of activities in general.
\end{abstract}

Keywords: Interorganizational relations. Competitive advantage. Higher education. 


\section{INTRODUÇÃO}

A competição no ambiente geral dos negócios tem sido enfrentada em diferentes contextos e por diferentes configurações organizacionais e interorganizacionais. A combinação de recursos internos e/ou externos para o alcance da vantagem competitiva é, em geral, uma tarefa que desafia o pragmatismo gerencial e o rigor acadêmico em busca de explicações efetivas às organizações e à academia. Via de regra, o foco orienta-se para a busca de retornos acima da média dos concorrentes, o que pode ser alcançado por meio da implementação de variadas estratégias de competição e/ou colaboração. No entanto, estratégias que envolvem relacionamentos interorganizacionais, como cooperação, associação, redes de empresas, entre outras, vêm ganhando força no cenário competitivo, o que já era preconizado por Dyer (1996a, 1996b, 1996c, 1997) e Jarillo (1988).

Ao longo do tempo, duas importantes abordagens emergiram em relação a fontes de retornos acima da média dos competidores (DYER; SINGH, 1998) ou a respeito da concepção da origem da vantagem competitiva (VASCONCELOS; CYRINO, 2000). A primeira, nominada como abordagem da estrutura da indústria e associada ao trabalho de Porter (1980), sugere que os retornos acima da média são principalmente uma função da atuação da empresa em uma indústria com características estruturais favoráveis, como poder de barganha, barreiras à entrada, entre outras. Como consequência, a indústria ou o setor é utilizado como unidade de análise relevante.

A segunda abordagem, denominada visão baseada em recursos da empresa (VBR), argumenta que o desempenho diferencial da empresa se deve fundamentalmente à sua heterogeneidade, em vez da estrutura da indústria, como defendido por Barney (1991) e Wernerfelt (1984). Nessa abordagem, a empresa é vista como a principal unidade de análise. Hoskisson et al. (1999) descrevem de forma detalhada a evolução teórica e metodológica do campo da gestão estratégica e a comparam às oscilações de um pêndulo, ora valorizando o ambiente externo como lócus para a formação da estratégia competitiva, ora apostando nos recursos internos como principais condutores da vantagem competitiva, ou, ainda, buscando o equilíbrio entre ambas as variáveis.

Embora essas duas abordagens tenham contribuído para a compreensão de como as empresas alcançam retornos acima da média, elas negligenciam o importante fato de que as (des)vantagens de uma empresa individual estão frequentemente relacionadas às (des)vantagens da rede de relacionamentos na qual ela está inserida. Logo, empresas que 


\section{RELAÇÕES INTERORGANIZACIONAIS COMO LÓCUS DE VANTAGEM COMPETITIVA: \\ MANIFESTAÇÕES EM INSTITUIÇÕES BRASILEIRAS DE EDUCAÇÃO SUPERIOR \\ DOI: http://dx.doi.org/10.5007/1983-4535.2017v10n2p274}

combinam recursos de maneira única podem perceber uma vantagem sobre os competidores que são incapazes ou não se engajam em relações interorganizacionais. Assim, as ligações idiossincráticas entre empresas podem ser uma fonte de rendas relacionais e vantagem competitiva. Esse olhar sugere que os recursos críticos de uma empresa podem estender os seus limites e podem ser incorporados em rotinas e processos entre empresas. Ou seja, o foco passa a estar nessas relações da rede/díade como importante unidade de análise para compreensão da vantagem competitiva (DYER; SINGH, 1998).

Nesse contexto, o objetivo deste artigo é analisar o desenvolvimento de relações interorganizacionais como fontes de vantagens competitivas. A partir disso, o objeto de estudo está focalizado em um consórcio de Instituições de Educação Superior (IES), denominado Consórcio das Universidades Comunitárias Gaúchas (COMUNG), localizado no sul do Brasil e formado por 15 Instituições Comunitárias de Educação Superior (ICES) com diferentes portes e regiões de atuação. Portanto, a seguinte questão orienta a investigação: como as relações interorganizacionais desenvolvidas por meio do COMUNG constituem-se em fontes de vantagem competitiva? Pretende-se com essa análise avançar na discussão teórica sobre relações interorganizacionais e vantagem competitiva, dado o contexto de país emergente e de setor com elevada regulação estatal.

A partir disso, os pressupostos teóricos que sustentam a discussão são explorados, centrados nos estudos sobre relações interorganizacionais e nas diferentes abordagens que tratam da vantagem competitiva. Na sequencia, o delineamento metodológico utilizado na pesquisa e as fontes de dados são detalhados e, logo, o caso do COMUNG é analisado. Por fim, o artigo expõe as conclusões e limitações da pesquisa.

\section{RELAÇÕES INTERORGANIZACIONAIS}

As relações interorganizacionais existem desde que há organizações que se relacionam umas com as outras. À medida que o estudo da economia, das sociedades e dos governos se estabeleceu e a relevância das organizações formais se tornou clara, estudiosos dessas áreas começaram a explorar alguns aspectos das relações interorganizacionais, o que forneceu as estruturas seminais para os estudos modernos dos fenômenos interorganizacionais em economia, sociologia e ciências políticas, como os de Marshall em 1923, Weber e Selznick em 1947. Apesar disso, a pedra fundamental para o estudo das relações interorganizacionais está relacionada ao desenvolvimento da teoria geral dos sistemas por von Bertalanffy, em 


\section{RELAÇÕES INTERORGANIZACIONAIS COMO LÓCUS DE VANTAGEM COMPETITIVA: \\ MANIFESTAÇÕES EM INSTITUIÇÕES BRASILEIRAS DE EDUCAÇÃO SUPERIOR \\ DOI: http://dx.doi.org/10.5007/1983-4535.2017v10n2p274}

1951, e sua aplicação a uma gama de problemas das ciências sociais por Boulding, em 1956 (CROPPER et al., 2014).

Em uma perspectiva evolucionária, uma síntese dos estudos envolvendo relações interorganizacionais pode ser encontrada em Johnsen, Lamming e Harland (2014), os quais apontam o desenvolvimento das investigações sobre relacionamentos, cadeias e redes desde os anos 1960 e 1970, onde as pesquisas em relação a cadeias interorganizacionais e, principalmente, redes, se encontravam em estágio inicial. Esses mesmos autores observam que, já na década de 1980, as pesquisas sobre cadeias expandiram significativamente a partir do conceito de cadeia de valor de Porter (1985) e, a partir dos anos 1990, há o início de uma grande onda de pesquisas sobre uma ampla gama de problemas relacionados a redes, como estratégias, funções e taxonomias.

Sob a ótica dos campos de estudo, as relações interorganizacionais têm sido pesquisadas por diferentes lentes teóricas, incluindo administração pública, marketing, economia e sociologia (WHETTEN, 1981), além da estratégia competitiva (JARILLO, 1988; THORELLI, 1986). Essa pluralidade de interesses deu origem a várias perspectivas distintas sobre relações interorganizacionais, como ecologia populacional, dependência de recursos, custos de transação, teoria institucional, entre outras (WILLIAMS, 2005). Essa variação está presente em vários trabalhos da área, como, por exemplo: a) o estudo de Boehe et al. (2011), o qual analisa os relacionamentos entre empresas e a capacidade de inovação como recursos e capacidades que explicam a propensão para a atividade exportadora; b) a pesquisa de Ma, Rhee e Yang (2013), acerca do impacto das fontes de poder, como propriedade e status, e a efetividade das relações interorganizacionais; c) o trabalho de Balestrin, Verschoore e Perucia (2014), que trata da visão relacional da estratégia em redes de cooperação empresarial, localizadas no Estado do Rio Grande do Sul (RS).

Conceitualmente, há dois pontos centrais que subjazem as pesquisas sobre relações interorganizacionais: as organizações e os relacionamentos entre elas. É a partir desses dois conceitos que todas as abordagens teóricas na pesquisa das relações interorganizacionais criam seus quadros distintivos de investigação, ou seja, um conjunto de dimensões descrevendo as organizações relacionadas e um conjunto de dimensões descrevendo a natureza dos relacionamentos por meio dos quais elas estão ligadas. Os diferentes tipos de pesquisas sobre relações interorganizacionais são diferenciados pelas formas específicas que caracterizam as organizações e seus inter-relacionamentos. Além disso, duas outras peças são 


\section{RELAÇÕES INTERORGANIZACIONAIS COMO LÓCUS DE VANTAGEM COMPETITIVA: \\ MANIFESTAÇÕES EM INSTITUIÇÕES BRASILEIRAS DE EDUCAÇÃO SUPERIOR \\ DOI: http://dx.doi.org/10.5007/1983-4535.2017v10n2p274}

importantes: o macro e/ou o micro contexto, no qual ambas as organizações e suas relações são imersas, e o processo por meio do qual as relações interorganizacionais são estabelecidas, mantidas, alteradas, dissolvidas e produzem resultados (CROPPER et al., 2014).

Devido aos benefícios potenciais das relações interorganizacionais, não é surpreendente que pesquisadores estejam interessados na busca por maneiras de tornar as colaborações menos complexas e políticas e mais previsíveis e eficientes. Em função disso, grande parte dos trabalhos nessa linha tem se focado nas diversas maneiras pelas quais as colaborações poderiam funcionar de forma mais eficiente. Por outro lado, perspectivas críticas de colaboração são mais céticas, por meio da indagação se as questões políticas podem ser superadas, presumindo que alguns parceiros estão mais interessados e adaptados a explorá-las (LOTIA; HARDY, 2014). O termo 'crítico' se refere ao trabalho que reconhece a existência de interesses divergentes, confirma as relações de poder raramente simétricas entre organizações e tenta incorporar as visões de grupos e indivíduos marginalizados em outras situações (ALVESSON; WILLMOTT, 1992).

Lotia e Hardy (2014) mostram que a literatura adota uma visão fundamentalmente funcionalista da colaboração, preocupada com a descoberta de soluções práticas para problemas práticos e baseada no pressuposto de que a colaboração é claramente benéfica quando trabalhada. Esses autores apontam ainda que, a partir dessa perspectiva, as colaborações são vistas como uma forma organizacional particular que pode ser delineada e administrada para aumentar a eficiência e a eficácia das organizações-membro individualmente e da própria colaboração como um todo.

Além disso, as consequências negativas da colaboração, como a injustiça, o abuso e a má utilização do poder, são, em grande parte, negligenciadas, ao passo que a ilusão de neutralidade contribui na justificativa das iniciativas de colaboração em bases racionais e funcionais. Enquanto esse corpo de trabalho fornece uma compreensão sobre como atores individuais podem administrar processos colaborativos complexos, falha ao não considerar que a colaboração pode ser por si só uma manobra política. Como resultado, questionamentos que interessam pesquisadores críticos passam ao largo, tais como se os benefícios são igualmente distribuídos, quais interesses prevalecem e se as colaborações podem ser prejudiciais a algumas organizações participantes (LOTIA; HARDY, 2014). A partir disso, diferentes abordagens sobre vantagem competitiva são apresentadas. 


\section{RELAÇÕES INTERORGANIZACIONAIS COMO LÓCUS DE VANTAGEM COMPETITIVA: \\ MANIFESTAÇÕES EM INSTITUIÇÕES BRASILEIRAS DE EDUCAÇÃO SUPERIOR \\ DOI: http://dx.doi.org/10.5007/1983-4535.2017v10n2p274}

\section{VANTAGEM COMPETITIVA}

As primeiras alusões à vantagem competitiva remetem à ideia de superioridade sobre os competidores, por meio de uma posição concorrencial forte e única (ANSOFF, 1965; BRITO; BRITO, 2012). Essa capacidade da empresa se destacar e superar o mercado fundamenta-se no modelo teórico de competição monopolística de Chamberlin, de 1933, que enfatiza a heterogeneidade e a diferenciação entre as empresas (BARNEY, 1986). A partir dos anos 1970, diversas correntes do pensamento econômico abordaram a questão da vantagem competitiva por meio de diferentes abordagens conceituais. No entanto, algumas das correntes contemporâneas não colocam verdadeiramente a questão das estratégias empresariais no centro de suas preocupações, como é o caso da economia neoclássica, da economia de custos de transação e da teoria da agência (VASCONCELOS; CYRINO, 2000). Enquanto a literatura de estratégia centra-se no estudo da superioridade concorrencial, a vantagem é um evento transitório e sem tanta importância em economia (WILLIAMSON, 1991).

À luz das estratégias empresariais, as teorias que tratam da vantagem competitiva podem ser divididas em dois eixos principais (VASCONCELOS; CYRINO, 2000). O primeiro eixo classifica os estudos em duas abordagens de acordo com sua concepção da origem da vantagem competitiva: a) as teorias que consideram a vantagem competitiva como um atributo de posicionamento, exterior à organização, derivado da estrutura da indústria, da dinâmica da concorrência e do mercado (outside-in); b) as teorias que tratam a performance superior como um fenômeno decorrente primariamente de características internas da organização (inside-out).

O segundo eixo, por sua vez, discrimina os estudos também em duas abordagens, segundo suas premissas sobre a concorrência: a) caracterizada pelos pesquisadores que possuem uma visão estrutural, essencialmente estática, da concorrência, fundada na noção de equilíbrio econômico; b) marcada pelos pesquisadores que enfocam os aspectos dinâmicos e mutáveis da concorrência, acentuando fenômenos como inovação, descontinuidade e desequilíbrio. A interseção desses dois eixos pode ser exemplificada nas seguintes correntes explicativas da vantagem competitiva: análise estrutural da indústria (fatores externos e estáticos); recursos e competências (fatores internos e estáticos); processos de mercado (fatores externos e dinâmicos); capacidades dinâmicas (fatores internos e dinâmicos).

Em geral, a discussão sobre as fontes que determinam a vantagem competitiva tem sido alvo de inúmeros e extensos debates entre teóricos das organizações e estratégia e pode 


\section{RELAÇÕES INTERORGANIZACIONAIS COMO LÓCUS DE VANTAGEM COMPETITIVA: \\ MANIFESTAÇÕES EM INSTITUIÇÕES BRASILEIRAS DE EDUCAÇÃO SUPERIOR \\ DOI: http://dx.doi.org/10.5007/1983-4535.2017v10n2p274}

ser sintetizada nas duas correntes principais: a) a que reconhece a determinação da vantagem competitiva de uma empresa, primariamente, a partir da sua adaptação ao ambiente externo, a qual vincula a vantagem à proteção das forças competitivas da indústria (PORTER, 1980); b) a que defende o desempenho superior da empresa como um fenômeno originado, primariamente, pelos recursos e capacidades da organização (BARNEY, 1991; WERNERFELT, 1984). Além disso, como observado, em muitos estudos o termo vantagem competitiva é empregado no sentido próximo a performance (CROOK et al., 2008), visto que vantagem competitiva é geralmente usada para descrever a performance relativa de rivais em um dado ambiente de mercado (PETERAF; BARNEY, 2003). No entanto, um problema comum nos estudos empíricos está relacionado ao design operacional do construto e à falta de clareza e diferenciação entre esses conceitos (BRITO; BRITO, 2012).

Embora essas correntes teóricas tenham contribuído de maneira significativa para a compreensão da formação e obtenção da vantagem competitiva, Dyer e Singh (1998) apontam que há um lócus para a criação de recursos existente entre os ambientes interno e externo às empresas, ou seja, entre as fronteiras da empresa e a estrutura da indústria. Esses autores abordam que a manifestação latente do processo de desenvolvimento de recursos, caracterizado pela interação da empresa com fornecedores, competidores, instituições de apoio à indústria, do governo e da sociedade civil, pode ser definido pelo conceito de relações interorganizacionais, e que o acesso a recursos idiossincráticos de outras empresas, indisponíveis aos seus concorrentes, constitui-se em fonte de vantagem competitiva relacional.

Essa abordagem, denominada visão relacional, foi originalmente desenvolvida a partir dos estudos de Dyer (1996a; 1996b, 1996c, 1997) por meio da análise de fabricantes japoneses e americanos de automóveis e seus fornecedores. Esses estudos revelaram que a fabricante japonesa Toyota desenvolvia relacionamentos mais próximos com seus fornecedores, os quais a diferenciava de seus competidores e, por consequência, consistiam em fontes de vantagem competitiva. Esses relacionamentos entre fabricante e fornecedores se baseavam em três pontos principais: a) proximidade física; b) investimentos em ativos físicos, como equipamentos e ferramentas; c) compartilhamento de conhecimentos por meio da interação humana.

A partir desses estudos, a tese central do trabalho de Dyer e Singh (1998) reside na afirmação de que uma díade ou rede de empresas pode desenvolver relacionamentos que 


\section{RELAÇÕES INTERORGANIZACIONAIS COMO LÓCUS DE VANTAGEM COMPETITIVA: \\ MANIFESTAÇÕES EM INSTITUIÇÕES BRASILEIRAS DE EDUCAÇÃO SUPERIOR \\ DOI: http://dx.doi.org/10.5007/1983-4535.2017v10n2p274}

resultam em vantagem competitiva sustentável. Duas outras importantes contribuições podem ser destacadas nesse trabalho: a) a colaboração entre empresas pode gerar rendas relacionais por meio de ativos específicos da relação, rotinas de compartilhamento de conhecimento, dotação de recursos complementares, e governança efetiva; b) a preservação das rendas relacionais, geradas por meio da colaboração efetiva entre empresas, ocorre pela identificação de mecanismos como: conectividade dos ativos interorganizacionais, escassez do parceiro, indivisibilidade dos recursos e o ambiente institucional.

Em termos gerais, empresas se envolvem em relações de cooperação em função dos ganhos potenciais da ação conjunta entre os agentes. No entanto, nem todas as formas de cooperação têm efeitos similares e são benéficas (BRITO; BRITO; HASHIBA, 2014). A obtenção de ganhos relacionais e vantagem competitiva depende diretamente do desenvolvimento e da manutenção de competências relacionais (LADO; PAURAJ; CHEN, 2011). O estudo de Brito, Brito e Hashiba (2014) aponta para o impacto negativo no crescimento da empresa por meio da resolução de problemas de forma compartilhada com consumidores e o impacto também negativo na sua rentabilidade por meio da resolução de problemas de forma compartilhada com fornecedores.

Vários outros estudos têm contribuído para o avanço das explicações sobre o temário da vantagem competitiva, como, por exemplo: a) o estudo de D’aveni, Dagnino e Smith (2010), o qual trata da geração de vantagens competitivas temporárias, por meio da revisão de uma série de trabalhos que discutem o caráter sustentável e duradouro das vantagens específicas da empresa; b) o trabalho de Brito e Brito (2012), que aborda uma discussão conceitual sobre vantagem competitiva e sua relação com o desempenho, baseado na criação de valor superior; c) a proposição do modelo de Lazzarini (2015), o qual estabelece a ligação entre política industrial (governamental) e vantagem competitiva, de forma mediada pelo acúmulo e movimentação rápida de recursos e capacidades locais e pela ação combinada de três condições: inserção em redes produtivas globais, especificidade geográfica e capacidade governamental.

A partir disso e dado o escopo deste artigo, adota-se como foco a abordagem de vantagem competitiva assentada no lócus das relações interorganizacionais, em detrimento das abordagens outside-in e inside-out. Outros estudos se embasaram nas vantagens relacionais entre organizações como foco de análise, como os de Manuj, Omar e 
Yazdanparast (2013), Viana, Barros e Añez (2014) e Vlaar, Van den Bosch e Volberda (2004).

\section{MÉTODO}

A partir de uma abordagem qualitativa, esta pesquisa centra-se em um estudo de caso único, com múltiplas unidades de análise (YIN, 2010). Essa técnica foi escolhida para compreender um fenômeno complexo e dependente do contexto (EISENHARDT, 1989; YIN, 2010). Como o COMUNG é composto por 15 ICES, adotou-se a proximidade geográfica, diferentes regiões de abrangência (ICES da região metropolitana e do interior do RS) e portes (número de alunos) como critérios de escolha das ICES pesquisadas, a fim de melhor evidenciar as relações interorganizacionais desenvolvidas pelo Consórcio. A partir desses critérios, seis ICES, além da presidência do COMUNG, foram pesquisadas no sentido de abranger as variadas experiências (vide Quadro 1).

\begin{tabular}{|c|c|c|c|}
\hline Instituição & Entrevistado(s) & Localização do(s) campus(i) & $\begin{array}{l}\text { Número de alunos } \\
\text { (base 2014) }\end{array}$ \\
\hline COMUNG & Presidente & & Cerca de 210 mil. \\
\hline $\begin{array}{l}\text { PUCRS }- \text { Pontifícia } \\
\text { Universidade Católica do Rio } \\
\text { Grande do Sul }\end{array}$ & $\begin{array}{l}\text { Pró-Reitor de } \\
\text { Pesquisa, Inovação } \\
\text { e Desenvolvimento }\end{array}$ & $\begin{array}{l}\text { Porto Alegre (campus } \\
\text { principal) e Viamão, na região } \\
\text { metropolitana de Porto Alegre. }\end{array}$ & Cerca de 30 mil. \\
\hline $\begin{array}{l}\text { UNIVATES }-\quad \text { Centro } \\
\text { Universitário UNIVATES }\end{array}$ & $\begin{array}{l}\text { Reitor e Pró-Reitor } \\
\text { de Administração }\end{array}$ & $\begin{array}{l}\text { Lajeado, no centro oriental do } \\
\text { RS e cerca de } 110 \mathrm{~km} \text { de Porto } \\
\text { Alegre. }\end{array}$ & Mais de 13 mil. \\
\hline $\begin{array}{l}\text { UNISC - Universidade de } \\
\text { Santa Cruz do Sul }\end{array}$ & $\begin{array}{l}\text { Pró-Reitor } \\
\text { Administração }\end{array}$ & $\begin{array}{l}\text { Santa Cruz do Sul, no centro } \\
\text { oriental do RS e cerca de } 60 \\
\mathrm{~km} \text { de Lajeado. }\end{array}$ & Mais de 12 mil. \\
\hline $\begin{array}{l}\text { UNILASALLE - } \quad \text { Centro } \\
\text { Universitário La Salle }\end{array}$ & $\begin{array}{l}\text { Pró-Reitor de } \\
\text { Desenvolvimento }\end{array}$ & $\begin{array}{lll}\text { Canoas, na } & \text { região } \\
\text { metropolitana de Porto } & \text { Alegre. }\end{array}$ & Mais de 8 mil. \\
\hline $\begin{array}{lcc}\text { UNIJUÍ } & - & \text { Universidade } \\
\text { Regional do Noroeste do } \\
\text { Estado do Rio Grande do Sul }\end{array}$ & Reitor & $\begin{array}{l}\text { Ijuí (campus principal), Santa } \\
\text { Rosa, Panambi e Três Passos, } \\
\text { no noroeste do RS. }\end{array}$ & Mais de 10 mil. \\
\hline $\begin{array}{l}\text { UNICRUZ - Universidade de } \\
\text { Cruz Alta }\end{array}$ & Reitora & $\begin{array}{l}\text { Cruz Alta, no noroeste do RS } \\
\text { e cerca de } 40 \mathrm{~km} \text { de Ijuí. }\end{array}$ & Cerca de 3 mil. \\
\hline
\end{tabular}

Quadro 1 ICES pesquisadas.

Fonte: Elaborado pelos autores.

Para a coleta de dados nessas ICES, diferentes fontes foram utilizadas por meio da documentação direta e indireta. Como fonte de documentação direta, a pesquisa se utilizou de entrevistas semiestruturadas com o Presidente do COMUNG e com os Reitores e/ou PróReitores das ICES selecionadas. Logo, sete entrevistas foram realizadas, cada uma com 
duração que variou de 40 minutos a 1 hora. O roteiro utilizado nas entrevistas se baseou em duas categorias de análise (vide Quadro 2).

Em relação à documentação indireta, a pesquisa se utilizou de informações já formalizadas pelo COMUNG e pelas ICES selecionadas em documentos internos, materiais de divulgação e nos seus próprios sítios institucionais. Ao associar as entrevistas realizadas com os dados secundários, observações e anotações do pesquisador, foi possível realizar a triangulação dos dados. Essa estratégia auxilia em análises mais completas, tendo em vista que leva em consideração mais de uma fonte de informação (FLICK, 2009). Utilizando tanto dados primários quanto secundários, a triangulação visou obter maior validade e confiabilidade ao se coletar dados em momentos diferentes, de fontes distintas ou por meio de mais de um único instrumento, no estudo de um mesmo fenômeno (STAKE, 1998).

\begin{tabular}{|c|l|l|}
\hline Categorias & \multicolumn{1}{|c|}{ Subcategorias } & \multicolumn{1}{|c|}{ Referências } \\
\hline \multirow{2}{*}{$\begin{array}{c}\text { Relações } \\
\text { interorganizacionais }\end{array}$} & $\begin{array}{l}\text { Ações colaborativas; abrangência e limites das } \\
\text { relações; confiança; poder; intensidade e densidade } \\
\text { das relações; macro e micro contexto; } \\
\text { relacionamentos interativos e não interativos; } \\
\text { consequências negativas da colaboração. }\end{array}$ & $\begin{array}{l}\text { Williams (2005); Lotia e } \\
\text { Hardy (2014). }\end{array}$ \\
\hline $\begin{array}{l}\text { Vantagem } \\
\text { competitiva } \\
\text { (relacional) }\end{array}$ & $\begin{array}{l}\text { Relacionamentos interorganizacionais e vantagens } \\
\text { relacionais; ativos específicos da relação; } \\
\text { compartilhamento de conhecimento; recursos } \\
\text { complementares. }\end{array}$ & $\begin{array}{l}\text { Dyer e Singh (1998); Dyer } \\
\text { Brito, Brito e Hashiba (2014); } \\
\text { Lado, Pauraj e Chen (2011). }\end{array}$ \\
\hline
\end{tabular}

Quadro 2 Categorias de análise utilizadas no roteiro de entrevistas.

Fonte: Elaborado pelos autores.

Para fins de análise dos dados, se utilizou a técnica de análise de conteúdo visando inferir conhecimentos por meio da geração de indicadores quantitativos ou não (BARDIN, 2011). A análise dos dados foi realizada por meio de resumos elaborados pelos pesquisadores, gravações das entrevistas, além dos materiais impressos e digitais. Os dados passaram por constante comparação (STRAUSS; CORBIN, 1990) entre a teoria e os resultados, visando avançar na discussão das vantagens competitivas relacionais.

\section{O CASO DO COMUNG}

Esta seção está organizada em duas partes, a fim de melhor contextualizar o caso em questão e os resultados da pesquisa. A primeira aborda o modelo comunitário praticado no Brasil e a caracterização do COMUNG e a segunda trata das vantagens relacionais usufruídas pelas ICES que integram o Consórcio. 


\subsection{O MODELO COMUNITÁRIO NO BRASIL E O COMUNG}

O modelo de educação superior brasileiro foi caracterizado pela dicotomia público $x$ privado até a promulgação da Constituição Federal de 1988, a qual reconheceu três modelos: a universidade pública estatal (federal, estadual ou municipal); a universidade comunitária ou pública não estatal; e a universidade particular (GUARESCHI, 2012), também conhecida como privada. No entanto, a ordem legal, representada pelo Código Civil, pela Lei de Diretrizes e Bases da Educação, pela legislação infraconstitucional e pelos atos administrativos em geral, continuou a reproduzir essa ultrapassada dicotomia na ausência de um marco jurídico apropriado, o que relegou, em geral, as ICES à condição de organizações privadas (LAZZARI; KOEHNTOPP; SCHMIDT, 2009). Após um longo período nessa condição, somente em 2013 essa disfunção foi corrigida por meio da Lei $\mathrm{N}^{\mathrm{o}} 12.881$, a qual define e qualifica as ICES, estabelece as suas prerrogativas e finalidades e regulamenta a cooperação entre o Estado e essas instituições.

Assim, as ICES foram legalmente reconhecidas pelas suas características, como: são constituídas juridicamente na forma de associações e fundações; seu patrimônio pertence a entidades da sociedade civil e/ou órgãos do poder público local/regional; não possuem fins lucrativos; seus resultados econômicos são integralmente reinvestidos na instituição; há transparência administrativa; a gestão é democrática; há participação da sociedade nos órgãos deliberativos; e há um profundo enraizamento na realidade regional (SCHMIDT; CAMPIS, 2009).

No Brasil, as ICES estão distribuídas por todo o território nacional, com destaque para o sul do país, onde há uma concentração significativa nos Estados do RS e de Santa Catarina (SC). O desenvolvimento do modelo comunitário no sul brasileiro pode ser representado no RS pelo COMUNG, formado por 15 ICES, e em SC pela Associação Catarinense das Fundações Educacionais (ACAFE), composta por 16 ICES. Em nível nacional, a Associação Brasileira de Universidades Comunitárias (ABRUC) representa o segmento, reunindo mais de 60 ICES do país.

Em suma, o COMUNG é um consórcio formado exclusivamente por ICES e possui cerca de 210 mil alunos de graduação e pós-graduação, o que representa aproximadamente $60 \%$ dos acadêmicos na educação superior do RS. As ICES que participam do COMUNG estão localizadas em diferentes regiões do RS, congregam mais de 40 campi universitários e abrangem mais de 380 municípios. As 15 ICES que o integram são: Universidade FEEVALE, 


\section{RELAÇÕES INTERORGANIZACIONAIS COMO LÓCUS DE VANTAGEM COMPETITIVA: \\ MANIFESTAÇÕES EM INSTITUIÇÕES BRASILEIRAS DE EDUCAÇÃO SUPERIOR \\ DOI: http://dx.doi.org/10.5007/1983-4535.2017v10n2p274}

Centro Universitário Metodista IPA, Pontifícia Universidade Católica do Rio Grande do Sul (PUCRS), Centro Universitário Franciscano (UNIFRA), Centro Universitário La Salle (Unilassalle), Universidade Católica de Pelotas (UCPel), Universidade de Caxias do Sul (UCS), Universidade de Cruz Alta (UNICRUZ), Universidade Regional do Noroeste do Estado do Rio Grande do Sul (UNIJUI), Universidade de Santa Cruz do Sul (UNISC), Universidade do Vale do Rio dos Sinos (UNISINOS), Centro Universitário UNIVATES, Universidade de Passo Fundo (UPF), Universidade da Região da Campanha (URCAMP) e Universidade Regional Integrada do Alto Uruguai e das Missões (URI).

Em função da abrangência geográfica e das áreas acadêmicas, é notório que essas ICES possuem sobreposição de oferta e disputam, muitas vezes, os mesmos mercados. No entanto, essas ICES também cooperam em determinadas atividades, como no fortalecimento da posição das ICES no contexto da educação superior brasileira e no poder político junto aos órgãos reguladores do governo federal, como Ministério da Educação (MEC), Coordenação de Aperfeiçoamento de Pessoal de Nível Superior (Capes) e Conselho Nacional de Desenvolvimento Científico e Tecnológico (CNPq).

Logo, esse convívio aparentemente dicotômico tem sido desenvolvido há cerca de 20 anos dentro do COMUNG, com períodos de maior ou menor engajamento entre as ICES que o integram, marcados pela própria dinâmica do consórcio, pelos recursos internos das ICES e pelas pressões setoriais, dos órgãos de regulação e do mercado. Em função desse histórico, este estudo enfoca os relacionamentos interorganizacionais desenvolvidos pelo COMUNG, no sentido de explorar teoricamente as evidências que condicionam a perpetuação desse arranjo interorganizacional e os elos que se constituem como fontes de vantagem competitiva.

\subsection{O COMUNG E AS VANTAGENS COMPETITIVAS RELACIONAIS}

Apesar da integração no mesmo consórcio, as ICES do COMUNG possuem diferenças significativas que impactam diretamente na competitividade, tais como: porte/tamanho, região de localização/abrangência, quantidade e qualidade dos cursos de graduação, programas de pós-graduação estrito senso e da produção científica, entre outras. Bengtsson e Kock (2000) apontam que a heterogeneidade dos recursos pode estimular a cooperação a fim de tornar essa relação uma fonte de vantagem competitiva.

De forma geral, os relacionamentos interorganizacionais desenvolvidos por meio do COMUNG podem ser categorizados em duas dimensões principais: a) ações administrativas, 


\section{RELAÇÕES INTERORGANIZACIONAIS COMO LÓCUS DE VANTAGEM COMPETITIVA: \\ MANIFESTAÇÕES EM INSTITUIÇÕES BRASILEIRAS DE EDUCAÇÃO SUPERIOR \\ DOI: http://dx.doi.org/10.5007/1983-4535.2017v10n2p274}

caracterizadas pela reunião de esforços e pelo poder de barganha frente aos aspectos políticos que regulam o setor de educação superior, junto a outras entidades, sindicatos, concorrentes e fornecedores de materiais e serviços, além do compartilhamento de experiências de gestão; b) ações acadêmicas, marcadas pelo compartilhamento de recursos e experiências de ensino, pesquisa e extensão, a fim de qualificar e diferenciar a atividade-fim das ICES. O Quadro 3 sintetiza as ações do Consórcio nessas duas dimensões, especificamente ou em ambas.

\begin{tabular}{|c|c|}
\hline Ações Administrativas & Ações Acadêmicas \\
\hline $\begin{array}{l}\text { - Movimentação política conjunta junto aos órgãos } \\
\text { reguladores do governo federal, como MEC, Capes, } \\
\text { CNPq, entre outros. } \\
\text { - Atuação conjunta a outras entidades regionais e } \\
\text { nacionais. } \\
\text { - Negociação conjunta com os sindicatos de } \\
\text { professores e técnico-administrativos. } \\
\text { - Compras conjuntas de materiais e softwares. }\end{array}$ & $\begin{array}{l}\text { - Associação para implementação conjunta de } \\
\text { programas de pós-graduação estrito senso (PPGs). } \\
\text { - Oferecimento de programa de formação continuada } \\
\text { de professores. } \\
\text { - Elaboração de editais internos de pesquisa. } \\
\text { - Convênio com universidades estrangeiras para } \\
\text { compartilhamento de experiências de alunos e } \\
\text { professores. }\end{array}$ \\
\hline \multicolumn{2}{|c|}{$\begin{array}{l}\text { - Compartilhamento de experiências gerenciais, procedimentos administrativos e de implementação e gestão } \\
\text { de cursos e programas acadêmicos. } \\
\text { - Realização de duas edições do Seminário do COMUNG para discussão das atividades de ensino, pesquisa, } \\
\text { extensão e gestão. } \\
\text { - Realização de duas edições do MBA em Gestão de IES para qualificação dos gestores, incluindo módulo } \\
\text { internacional em universidades europeias. } \\
\text { - Acordos tácitos de competição. }\end{array}$} \\
\hline
\end{tabular}

Quadro 3 Ações do COMUNG como fontes de vantagens competitivas relacionais.

Fonte: Dados coletados na pesquisa.

Como se trata de um setor significativamente regulado pela esfera federal, a movimentação política em bloco proporciona uma série de benefícios que dificilmente seriam possíveis em tentativas particulares. Esses benefícios se retratam no acompanhamento direto dos processos de formulação de políticas para o setor e na participação ativa em determinados assuntos da área, o que possibilita a melhor preparação para o enfrentamento de novas regulamentações e a atualização de forma mais rápida. Além disso, o próprio tamanho do país e o consequente número de IES apontam para a dificuldade de atuação individual junto aos órgãos reguladores e orientam para a melhor efetividade por meio da atuação conjunta, como a participação ativa e o apoio na construção e implementação, pelo governo federal, de programas de financiamento estudantil (ex.: Fundo de Financiamento Estudantil - FIES) e de bolsas de estudos parciais e integrais (ex.: Programa Universidade para Todos - PROUNI).

Em função dessa proximidade, da forma de atuação e da qualidade acadêmica das ICES do COMUNG, a participação junto aos órgãos federais é ainda mais estreita em determinadas matérias, como, por exemplo, no caso do FIES, o qual passou por uma 


\section{RELAÇÕES INTERORGANIZACIONAIS COMO LÓCUS DE VANTAGEM COMPETITIVA: \\ MANIFESTAÇÕES EM INSTITUIÇÕES BRASILEIRAS DE EDUCAÇÃO SUPERIOR \\ DOI: http://dx.doi.org/10.5007/1983-4535.2017v10n2p274}

reformulação geral a partir de 2010 e utilizou uma das ICES do COMUNG, a UNISC, como projeto piloto nacional para o desenvolvimento do novo programa. Além disso, a participação de reitores do COMUNG na direção da ABRUC tem dado notoriedade ao Consórcio e oportunizado o diálogo aproximado com autoridades educacionais do país.

Nesse mesmo campo político, outras ações com participação direta do COMUNG, em conjunto com outras entidades, como ABRUC, ACAFE, Associação Brasileira de Instituições Educacionais Evangélicas (ABIEE), Associação Nacional de Educação Católica do Brasil (ANEC), entre outras, reforçaram o seu poder de barganha junto ao governo federal e se refletiram em benefícios tangíveis para as suas ICES, com destaque para: a) participação ativa na construção e aprovação da Lei $\mathrm{N}^{\mathrm{o}} 12.688$, de 2012, que institui o plano de recuperação tributária (Programa de Estímulo à Reestruturação e ao Fortalecimento das Instituições de Educação Superior - PROIES), o qual viabilizou a renegociação de dívidas tributárias federais, por meio do pagamento em espécie ou bolsas de estudo; b) participação ativa na construção e aprovação da Lei $\mathrm{N}^{\mathrm{o}} 12.881$, de 2013, que superou a dicotomia público $x$ privado, por meio do reconhecimento efetivo do status comunitário de IES, e regulamentou o acesso a editais de órgãos governamentais de fomento direcionados às instituições públicas, o recebimento de recursos orçamentários do poder público para o desenvolvimento de atividades de interesse público, entre outras prerrogativas. Essas leis possibilitaram a reorganização administrativa e financeira de algumas ICES do COMUNG, a ampliação das opções de fomento para as atividades de pesquisa e extensão e, especialmente, o reconhecimento das características das ICES, distinguindo-as claramente das IES particulares/privadas.

$\mathrm{Na}$ interseção das ações administrativas e acadêmicas, o compartilhamento de experiências entre as coirmãs do COMUNG é um ponto central das vantagens relacionais, como abordado por Dyer e Singh (1998). Duas ações são representativas dessa cooperação acadêmica e administrativa: a) os seminários realizados pelo COMUNG, para a discussão de temas latentes nas atividades de ensino, pesquisa, extensão e gestão, com a participação de experientes profissionais no contexto nacional e internacional; b) o curso de MBA em Gestão de IES, proposto pelo COMUNG e coordenado pela UCS, o qual oportuniza trocas formais e informais de experiências entre os gestores, por meio de módulos temáticos concentrados em períodos semanais e de forma rotativa entre as ICES anfitriãs. Esses aspectos possuem convergência com os benefícios constatados por Muijs et al. (2011), que incluem a 


\section{RELAÇÕES INTERORGANIZACIONAIS COMO LÓCUS DE VANTAGEM COMPETITIVA: \\ MANIFESTAÇÕES EM INSTITUIÇÕES BRASILEIRAS DE EDUCAÇÃO SUPERIOR \\ DOI: http://dx.doi.org/10.5007/1983-4535.2017v10n2p274}

transferência de experiências profissionais, práticas e políticas entre as instituições na capacidade de preencher lacunas organizacionais e adquirir resiliência a mudanças externas.

No campo administrativo, essas ICES trocam, com frequência, experiências relacionadas a operação no setor, envolvendo inúmeras atividades de cunho estratégico ou operacional, como: decisões de terceirização; negociação sindical; resolução de problemas de segurança nas instalações das ICES; aquisição de softwares ERPs (Enterprise Resource Planning) já utilizados por outras coirmãs; operacionalização de sistemas de bolsas, financiamento estudantil e outros próprios do setor; estrutura e programação das TVs universitárias; resolução de problemas de tecnologia de informação; entre outras.

Essas trocas de informações e experiências também ocorrem de forma similar no campo acadêmico entre as ICES do COMUNG. Nas atividades de ensino, há inúmeras interações entre gestores e docentes a fim de acelerar o aprendizado e evitar caminhos desnecessários. Nesse sentido, as ICES com experiência em determinada área auxiliam aquelas que são novatas, como, por exemplo, a experiência compartilhada pela UNISC na implantação do Curso de Medicina, a partir do método da aprendizagem baseada em problemas. Essa experiência da UNISC, envolvendo aspectos da estrutura física e pedagógica, foi, inclusive, utilizada pela UNIVATES, localizada próxima geograficamente, e que está em fase de implantação desse curso.

Outros relacionamentos interorganizacionais, de maior envergadura e desenvolvidos por ICES do COMUNG, estão representados pelo oferecimento de forma associada de PPGs. Essa atividade envolve uma aproximação maior entre as ICES, especialmente entre os docentes da área na qual o programa é ofertado, e uma colaboração de médio a longo prazo. Para as ICES, isso potencializa recursos complementares, como indicado por Dyer e Singh (1998), e pode trazer inúmeros benefícios, como: fixação de recursos escassos (nesse caso, os docentes); condicionamento para o (ou manutenção do) status de universidade, sob a exigência de um número mínimo de PPGs; reforço das atividades da graduação; desenvolvimento de pesquisas conjuntas; entre outros. São exemplos desse tipo de associação o Mestrado em Atenção Integral à Saúde, desenvolvido pela UNICRUZ e UNIJUÍ, e o Doutorado em Administração, oferecido pela PUCRS e UCS.

Ainda na área de ensino, a cooperação entre as ICES do COMUNG se estende para o processo continuado de formação de professores, a fim de qualificar e inovar a prática pedagógica. Nessa linha, o Consórcio atua em duas frentes: uma desenvolvida para 


\section{RELAÇÕES INTERORGANIZACIONAIS COMO LÓCUS DE VANTAGEM COMPETITIVA: \\ MANIFESTAÇÕES EM INSTITUIÇÕES BRASILEIRAS DE EDUCAÇÃO SUPERIOR \\ DOI: http://dx.doi.org/10.5007/1983-4535.2017v10n2p274}

professores em geral, que já contou com especialistas finlandeses tratando da pedagogia inovativa em uma de suas edições, e outra especificamente para professores da área das Engenharias. Além do ensino, o COMUNG também tem fomentado a formação de linhas conjuntas de pesquisa, por meio da publicação de editais envolvendo pesquisadores de, no mínimo, três ICES, e a internacionalização do Consórcio pela mobilidade acadêmica com IES do exterior, como, por exemplo, o convênio com o grupo alemão UAS7 - Seven Universities of Applied Sciences.

Além desse ambiente de cooperação, a competição entre as ICES do COMUNG ocorre naturalmente pela disputa de alunos, elevação da qualidade das atividades e do conceito dos cursos, especialmente entre aquelas que estão próximas fisicamente. Nesse sentido, não há acordos formais estabelecidos entre essas instituições, tanto nas áreas geográficas de atuação quanto nas áreas acadêmicas ou do conhecimento. No entanto, essa competição respeita vários elementos que suavizam as relações de mercado, por meio da observância das áreas geográficas de atuação e da concorrência não predatória ou agressiva.

Esses elementos são convencionados ao longo do tempo e construídos tacitamente entre as ICES, baseados nas relações de confiança, como apontado por Williams (2005). Gold, Seuring e Beske (2010) consideram a confiança um recurso relacional valioso, que não pode ser comercializado e de difícil imitação pelos concorrentes. Para essa relação, pode ser utilizado o termo "competição civilizada", o qual exprime um conjunto de regras que permitem fortalecer a posição competitiva do grupo (MUIJS; RUMYANTSEVA, 2014). Além disso, as ICES de menor porte são favorecidas por uma moderação dessa competição em relação às ICES detentoras de mais recursos.

Esses acordos salvaguardam parcialmente o território de atuação das ICES do COMUNG. Isso é mais notório entre as IES que estão localizadas no interior do RS, pois dificilmente uma ICES que pertence ao COMUNG vai se estabelecer em uma cidade onde está localizada uma coirmã. Essa é uma importante vantagem relacional para as ICES, dado que há uma dependência significativa da receita dos cursos de graduação e a maior parte dos alunos na educação superior brasileira possui um perfil "trabalhador", o que torna o públicoalvo relativamente regionalizado. Bleeke e Ernst (2001) apontam que a cooperação evita a disputa direta com concorrentes, delimitando o conflito com base em um acordo cooperativo que possibilite manter a sustentabilidade da vantagem competitiva adquirida ou que favoreça 


\section{RELAÇÕES INTERORGANIZACIONAIS COMO LÓCUS DE VANTAGEM COMPETITIVA: \\ MANIFESTAÇÕES EM INSTITUIÇÕES BRASILEIRAS DE EDUCAÇÃO SUPERIOR \\ DOI: http://dx.doi.org/10.5007/1983-4535.2017v10n2p274}

a geração de valor. Isso também pode ser estendido além-muros do Consórcio, pois a atuação cooperativa das ICES eleva as barreiras a potenciais entrantes na educação superior do RS.

Em suma, essas ações administrativas e acadêmicas compartilhadas entre as ICES do COMUNG representam fontes de vantagens relacionais, dado a dificuldade ou impossibilidade de outras IES acessarem os mesmos benefícios. Cabe destacar ainda que essas ações não são homogêneas entre todas as ICES do Consórcio, havendo interesses variados nas relações de cooperação.

\section{CONCLUSÃO}

Os relacionamentos interorganizacionais têm se constituído como importante lócus de vantagem competitiva, de forma complementar às tradicionais abordagens outside-in e insideout. Localizadas entre as fronteiras da empresa ou organização e a estrutura da indústria, essas relações revelam fontes de vantagem competitiva que não estão disponíveis internamente nas organizações e tampouco na estrutura da indústria. São, de fato, elos intermediários que podem gerar vantagens particulares e indisponíveis àquelas que não participam da díade ou rede. Logo, a posição de insider ou outsider pode ser determinante no alcance de uma determinada vantagem competitiva.

À luz da abordagem das vantagens relacionais de Dyer e Singh (1998), as ações realizadas por meio do COMUNG evidenciam isso claramente. A utilização de boa parte dessas ações como fonte de vantagem competitiva só é possível para um insider, como o aprendizado por meio do compartilhamento de experiências administrativas e acadêmicas e a salvaguarda das áreas geográficas de competição. Como abordado por Dyer e Singh (1998), uma estratégia efetiva da visão relacional pode estar em empresas que compartilham sistematicamente conhecimentos valiosos com os parceiros da aliança e aceitam cientemente algumas formas de competição, a fim de acessar o acúmulo de experiências que residem no parceiro.

Portanto, essas ações notadamente têm sido fontes de vantagem competitiva para as ICES do COMUNG, por meio da redução e do compartilhamento de custos, do aprendizado de forma mais rápida e por caminhos mais profícuos, e da qualificação e diferenciação das atividades em geral. No entanto, a realização de pesquisa no temário da vantagem competitiva é, de certa forma, um emaranhado de relações complexas. Neste estudo, por exemplo, não há a mensuração quantitativa da vantagem competitiva, dado o foco estabelecido e a dificuldade 
de isolar determinadas ações realizadas por meio do COMUNG das demais ações individuais de cada ICES.

Por outro lado, novas avenidas de pesquisa podem ser visualizadas, por meio de inéditas relações teóricas e/ou constatações empíricas. À exemplo das conclusões de Hoskisson et al. (1999), a visão relacional se beneficia das abordagens anteriores de pesquisa, outside-in e inside-out, e contribui para o maior domínio da área, conduzindo os pesquisadores a uma gama mais ampla de perguntas no temário geral da vantagem competitiva. Além deste estudo, outras teorias também podem contribuir na explicação do caso em questão, como, por exemplo, a perspectiva co-evolucionária (LEWIN; VOLBERDA, 1999), a qual pode utilizar de forma sistêmica as três abordagens mencionadas.

\section{REFERÊNCIAS}

ALVESSON, M.; WILLMOTT, H. On the idea of emancipation in management and organization studies. Academy of Management Review, v. 17, n. 3, p. 432-464, 1992.

ANSOFF, H.I. Corporate Strategy: an analytic approach to business policy for growth and expansion. New York: McGraw-Hill, 1965.

BALESTRIN, A.; VERSCHOORE, J.R.; PERUCIA, A. A visão relacional da estratégia: evidências empíricas em redes de cooperação empresarial. BASE - Revista de Administração e Contabilidade da Unisinos, v. 11, n. 1, p. 47-58, 2014.

BARDIN, L. Análise de Conteúdo. São Paulo: Edições 70, 2011.

BARNEY, J.B. Types of competition and the theory of strategy: toward an integrative framework. Academy of Management Review, v. 11, n. 4, p. 791-800, 1986.

. Firm resources and sustained competitive advantage. Journal of Management, v. 17, n. 1, p. 99-120, 1991.

BENGTSSON, M.; KOCK, S. "Coopetition" in business networks - to cooperate and compete simultaneously. Industrial Marketing Management, v. 29, p. 411-426, 2000.

BLEEKE, J.; ERNST, D. Colaborando para competir. In: MINTZBERG, H.; QUINN, J.B. O Processo da Estratégia. 3. ed. Porto Alegre: Bookman, 2001.

BOEHE, D.M. et al. Papel das relações interorganizacionais e da capacidade de inovação na propensão para exportar. REAd - Revista Eletrônica de Administração, v. 17, n.1, p. 86$116,2011$. 
BRITO, L.A.L.; BRITO, E.P.Z.; HASHIBA, L.H. What type of cooperation with suppliers and customers leads to superior performance? Journal of Business Research, v. 67, p. 952$959,2014$.

BRITO, R.P.; BRITO, L.A.L. Vantagem competitiva e sua relação com o desempenho - uma abordagem baseada em valor. RAC - Revista de Administração Contemporânea, v. 16, n. 3, p. 360-380, 2012.

CROOK, T.R. et al. Strategic resources and performance: a meta-analysis. Strategic Management Journal, v. 29, p. 1141-1154, 2008.

CROPPER, S. et al. Introdução às relações interorganizacionais. In: Relações Interorganizacionais da Oxford. Porto Alegre: Bookman, 2014. . Handbook de

D'AVENI, R.A.; DAGNINO, G.B.; SMITH, K.G. The age of temporary advantage. Strategic Management Journal, v. 31, p. 1371-1385, 2010.

DYER, J.H. Specialized supplier networks as a source of competitive advantage: evidence from the auto industry. Strategic Management Journal, v. 17, n. 4, p. 271-291, 1996a.

. Does governance matter? Keiretsu alliances and asset specificity as sources of Japanese competitive advantage. Organization Science, v. 7, n. 6, p. 649-666, $1996 \mathrm{~b}$.

. How Chrysler created an American keiretsu. Harvard Business Review, v. 74, n. 4, p. 42-56, 1996c.

. Effective interfirm collaboration: how firms minimize transaction costs and

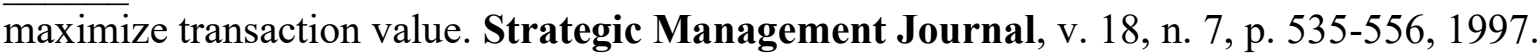

; SINGH, H. The relational view: cooperative strategy and sources of interorganizational competitive advantage. The Academy of Management Review, v. 23, $\mathrm{n}$. 4, p. 660-679, 1998.

EISENHARDT, K.M. Building theories from case study research. Academy of Management Review, v. 14, n. 4, p. 532-550, 1989.

FLICK, U. Desenho da Pesquisa Qualitativa. Porto Alegre: Artmed, 2009.

GOLD, S.; SEURING, S.; BESKE, P. Sustainable supply chain management and interorganizational resources: a literature review. Corporate Social Responsibility and Environmental Management, v. 17, n. 4, p. 230-245, 2010.

GUARESCHI, A. Universidade Comunitária: uma experiência inovadora. Passo Fundo: Berthier; Aldeia Sul, 2012.

HOSKISSON, R.E. et al. Theory and research in strategic management: swings of a pendulum. Journal of Management, v. 25, n. 3, p. 417-456, 1999. 
JARILLO, J.C. On strategic networks. Strategic Management Journal, v. 9, p. 31-41, 1988.

JOHNSEN, T.E.; LAMMING, R.C.; HARLAND, C.M. Relacionamentos

interorganizacionais, cadeias e redes: uma perspectiva de suprimentos. In: CROPPER, S. et al. Handbook de Relações Interorganizacionais da Oxford. Porto Alegre: Bookman, 2014

LADO, A.A.; PAURAJ, A.; CHEN, I.J. Costumer focus, supply-chain relational capabilities and performance. The International Journal of Logistics Management, v. 22, n. 2, p. $202-$ $221,2011$.

LAZZARI, N.J.; KOEHNTOPP, P.I; SCHMIDT, J.P. Apresentação. In: SCHMIDT, J.P. Instituições Comunitárias: instituições públicas não-estatais. Santa Cruz do Sul: EDUNISC, 2009.

LAZZARINI, S.G. Strategizing by the government: can industrial policy create firm-level competitive advantage? Strategic Management Journal, v. 36, p. 97-112, 2015.

LEWIN, A.Y.; VOLBERDA, H. Prolegomena on coevolution: a framework for research on strategy and new organizational forms. Organization Science, v. 10, n. 5, p. 519-534, 1999.

LOTIA, H.; HARDY, C. Perspectivas críticas de colaboração. In: CROPPER, S. et al. Handbook de Relações Interorganizacionais da Oxford. Porto Alegre: Bookman, 2014.

MA, D.; RHEE, M.; YANG, D. Power source mismatch and the effectiveness of interorganizational relations: the case of venture capital syndication. Academy of Management Journal, v. 56, n. 3, p. 711-734, 2013.

MANUJ, I.; OMAR, A.; YASDANPARAST, A. The quest for competitive advantage in global supply chains: the role of inteorganizational learning. Transportation Journal, v. 52, n. 4, p. 463-492, 2013.

MUIJS, D. et al. Collaboration and Networking in Education. Dordrecht: Springer, 2011.

; RUMYANTSEVA, N. Coopetition in education: collaborating in a competitive environment. Journal of Educational Change, v. 15, n. 1, p. 1-18, 2014.

PETERAF, M.A.; BARNEY, J.B. Unraveling the resource-based tangle. Managerial and Decision Economics, v. 24, n. 4, p. 309-323, 2003.

PORTER, M.E. Competitive Strategy. New York: Free Press, 1980.

Competitive Advantage: creating and sustaining superior performance. New York: Free Press, 1985.

SCHMIDT, J.P.; CAMPIS, L.A.C. As instituições comunitárias e o novo marco jurídico do público não estatal. In: SCHMIDT, J.P. Instituições Comunitárias: instituições públicas nãoestatais. Santa Cruz do Sul: EDUNISC, 2009. 
STAKE, R. Case studies. In: DENZIN, N.; LINCOLN, Y. (eds.). Strategies of Qualitative Inquiry. Thousand Oaks, London, New Delhi: Sage, 1998.

STRAUSS, A.; CORBIN, J. Basics of Qualitative Research: Grounded theory procedures and techniques. Newbury Park, CA: Sage, 1990.

THORELLI, H.B. Networks: between markets and hierarchies. Strategic Management Journal, v. 7, p. 37-51, 1986.

VASCONCELOS, F.C.; CYRINO, Á.B. Vantagem competitiva: os modelos teóricos atuais e a convergência entre estratégia e teoria organizacional. RAE - Revista de Administração de Empresas, v. 40, n. 4, p. 20-37, 2000.

VIANA, F.L.E.; BARROS, J.P., Neto; AÑEZ, M.E.M. Gestão da cadeia de suprimento e vantagem competitiva relacional nas indústrias têxtil e de calçados. Gestão \& Produção, v. 21, n. 4, p. 836-852, 2014.

VLAAR, P.W.L.; VAN DEN BOSCH, F.A.J.; VOLBERDA, H.W. On the relation between information technology and interorganizational competitive advantage: a competence perspective. Advances in Apllied Business Strategy, v. 8, p. 45-68, 2004.

WERNERFELT, B. A resource-based view of the firm. Strategic Management Journal, v. 5, p. 171-180, 1984.

WHETTEN, D.A. Interorganizational relations: a review of the field. Journal of Higher Education, v. 52, n. 1, p. 1-28, 1981.

WILLIAMS, T. Cooperation by design: structure and cooperation in interorganizational networks. Journal of Business Research, v. 58, p. 223-231, 2005.

WILLIAMSON, O.E. Strategizing, economizing, and economic organization. Strategic Management Journal, v. 12, p. 75-94, 1991.

YIN, R.K. Estudo de Caso: planejamento e métodos. 4. ed. Porto Alegre: Bookman, 2010. 